ARTICLE

https://doi.org/10.1038/s41467-019-12168-8

\title{
From spinodal decomposition to alternating layered structure within single crystals of biogenic magnesium calcite
}

\author{
Eva Seknazi ${ }^{1}$, Stas Kozachkevich¹, Iryna Polishchuk1, Nuphar Bianco Stein ${ }^{1}$, Julie Villanova², \\ Jussi-Petteri Suuronen (10 ${ }^{2}$, Catherine Dejoie ${ }^{2}$, Paul Zaslansky ${ }^{3}$, Alex Katsman ${ }^{1} \&$ Boaz Pokroy ${ }^{1}$
}

As organisms can form crystals only under ambient conditions, they demonstrate fascinating strategies to overcome this limitation. Recently, we reported a previously unknown biostrategy for toughening brittle calcite crystals, using coherently incorporated Mg-rich nanoprecipitates arranged in a layered manner in the lenses of a brittle star, Ophiocoma wendtii. Here we propose the mechanisms of formation of this functional hierarchical structure under conditions of ambient temperature and limited solid diffusion. We propose that formation proceeds via a spinodal decomposition of a liquid or gel-like magnesium amorphous calcium carbonate (Mg-ACC) precursor into Mg-rich nanoparticles and a Mg-depleted amorphous matrix. In a second step, crystallization of the decomposed amorphous precursor leads to the formation of high-Mg particle-rich layers. The model is supported by our experimental results in synthetic systems. These insights have significant implications for fundamental understanding of the role of Mg-ACC material transformation during crystallization and its subsequent stability.

\footnotetext{
${ }^{1}$ Department of Materials Science and Engineering and the Russel Berrie Nanotechnology Institute, Technion-Israel Institute of Technology, 32000 Haifa, Israel. ${ }^{2}$ ESRF-The European Synchrotron Radiation Facility, CS 40220, 38043 Grenoble Cedex 9, France. ${ }^{3}$ Department for Restorative and Preventive Dentistry, Centrum für Zahn-, Mund- und Kieferheilkunde, Charité-Universitätsmedizin Berlin, 14197 Berlin, Germany. Correspondence and requests for materials should be addressed to B.P. (email: bpokroy@technion.ac.il)
} 
B iominerals are minerals that originate from biological processes. Although formed under ambient conditions and from only a limited variety of elements, biominerals can exhibit outstanding properties related to their specific functions ${ }^{1-6}$. For example, the calcitic teeth of the sea urchin are composed of brittle calcite, yet they exhibit mechanical properties optimized to serve their grinding function ${ }^{3,4}$. Likewise, the lenses of the brittle star possess optimized optical properties despite being composed of calcite, a birefringent material ${ }^{5}$. Considerable research has focused on these biominerals from the perspective of materials science, in an attempt to understand the specific compositions and structures responsible for their superior properties $^{7-9}$, and to learn strategies that can be applied in the design of novel synthetic materials ${ }^{10-15}$

Research on marine biominerals has highlighted recurrent strategies for enhancement of mechanical properties. Common strategies include the use of hierarchical structures ${ }^{8,10}$, exploiting the presence of intra- and inter-crystalline organic molecules $1,2,16$, and substitution of $\mathrm{Mg}$ for $\mathrm{Ca}$ in calcitic biominerals $3,4,6,9$. Recently, Polishchuk et al. uncovered a strategy that involves a complex distribution of $\mathrm{Mg}$ in calcite, and which acts as a toughening mechanism in the lenses of the brittle star Ophiocoma wendtii 6 .

The brittle star is an echinoderm that possesses several skeletal parts ${ }^{17}$, including lenses, spicules, teeth, and arm vertebrae, which, respectively, serve its visual system ${ }^{5}$, locomotor system, oral system, and act as joints ${ }^{18}$. A study of the lens nanostructure showed that each lens comprises a single crystal composed of two coherent Mg-calcite phases: a phase comprising nanoparticles of high- $\mathrm{Mg}$ calcite ( $\sim 5 \mathrm{~nm}$ diameter, $\sim 40 \mathrm{~mol} \% \mathrm{MgCO}_{3}$ ), coherently incorporated in a phase consisting of a poorer $\mathrm{Mg}$-calcite matrix $\left(\sim 13 \mathrm{~mol} \% \mathrm{MgCO}_{3}\right)^{6}$. It was further shown that the nanoprecipitates are not dispersed homogeneously within the single-crystalline lenses, but are organized in layers, parallel to the surface, that are alternately richer and poorer in Mg-nanoinclusions ${ }^{6}$. These two nanostructural features-the existence of coherent $\mathrm{Mg}$-rich nanoinclusions and their layered distribution-improve lens toughness (increasing it by three-fold compared to geological calcite $)^{6}$. Together, the existing coherence and the lattice mismatch between the Mg-rich nanoinclusions and their surrounding matrix induce compressive stresses in the matrix, creating a prestressed material with consequently improved hardness and fracture toughness ${ }^{6}$. Moreover, the parallel arched layers within the lenses create a structure with

a

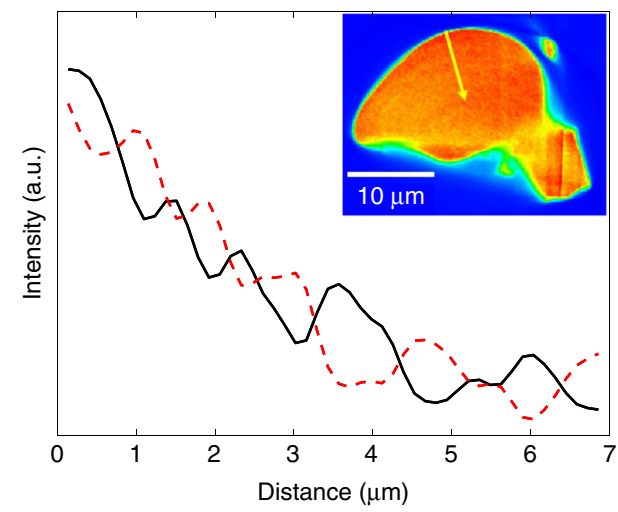

alternating compressive stresses and elastic modulus, promoting crack deflection and thus further enhancing their toughness ${ }^{6}$. As pointed out by Duffy ${ }^{19}$, this discovered strategy may represent the solution to the dual need of optimizing the mechanical function of the lens without compromising its optical function.

In this work, utilizing synchrotron radiation and electron microscopy techniques, we characterized the layered structure of the brittle star lenses and spicules. We also addressed the remaining fundamental questions concerning the mechanisms of formation of this complex, highly functional nanostructured material under ambient conditions. We propose a formation scenario of the brittle star lens nanostructure, involving spinodal decomposition of the amorphous supersaturated precursor followed by crystallization from a single nucleation event. To support the proposed biological route of formation we present experimental results that indicate that in synthetic $\mathrm{Mg}$ amorphous calcium carbonate (Mg-ACC) a spinodal decomposition indeed precedes crystallization of higher and lower $\mathrm{Mg}$-calcite phases. We also show that organics suppress the spinodal decomposition and that most probably the paucity of organics present in the brittle star's mineralized tissue allows it to occur in this system. Unraveling the mechanism of formation of the brittle star's calcite biomineral has important implications for understanding the role of the Mg-ACC material prior to and during crystallization and for the success of synthetically growing various single crystals at ambient temperatures, which is not possible as yet.

\section{Results}

The layered structure. Our previous study of the nanostructure of brittle star lenses provided strong evidence for the presence of arched layers oriented parallel to the lens surface ${ }^{6}$. Among others, small-angle X-ray scattering (SAXS) measurements indicated a layered distribution of few $\mathrm{nm}$-sized features (periodicity of $\sim 2.5-16.5 \mathrm{~nm}$ ). In quest to further understand what these layers are comprised of, we performed additional characterization utilizing several techniques. Firstly, we used synchrotron X-ray fluorescence (XRF) tomography obtained by a nanobeam in the synchrotron to map the elemental distribution of the lenses. A typical reconstruction map of Ca distribution (Fig. 1a, inset) and the corresponding intensity line plots (Fig. 1a) reveal intensity oscillations of this specific element.

Oscillation of the Ca signal correlates well with the existence of alternating $\mathrm{Ca}$-depleted and $\mathrm{Ca}$-rich layers beneath the lens

b

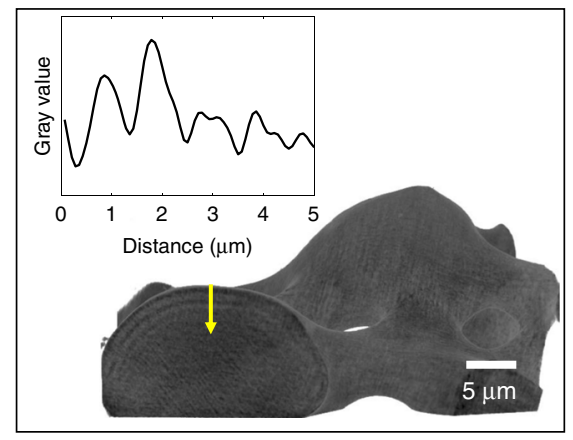

Fig. 1 The layered structure revealed by tomography. a XRF tomography reconstruction slice across a lens (pixel size is $150 \mathrm{~nm}$, red areas correspond to higher $\mathrm{Ca}$ content). Corresponding intensity line plot taken from the lens surface towards the center of the bulk (marked by a yellow arrow), shows the measured oscillations of the calcium signal (black) compared with theoretical schematic oscillations of the magnesium content (dashed red), perpendicular to the sample surface. $\mathbf{b}$ Typical nano-HT reconstructed dorsal arm plate (DAP) part, showing oscillations in mass-density (pixel size is $35 \mathrm{~nm}$ ).

Corresponding intensity line plot taken from the lens surface towards the center of the bulk (marked by a yellow arrow) 

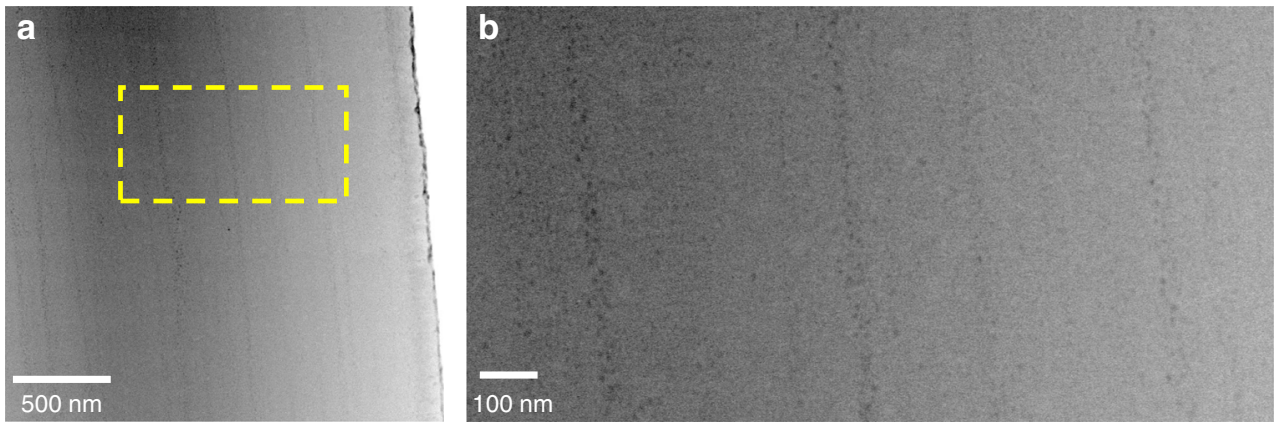

Fig. 2 The layered structure is caused by a layered distribution of high-Mg nanoparticles. a HRTEM (HAADF-STEM mode) of a FIB-cut lens lamella (cut perpendicularly to a lens surface-the lens surface can be seen at the right edge of the image) showing the existence of light (dark) nanoparticles distributed in layers parallel to the surface. $\mathbf{b}$ Zoom-in corresponding to the yellow square in (a)

surface. We could not obtain a signal for $\mathrm{Mg}$ due to energy detection restriction (the energy of the $\mathrm{K}$-alpha radiation is too low and absorbed by air surrounding the sample during measurement). Nevertheless, since $\mathrm{Mg}$ substitutes for $\mathrm{Ca}$ in a calcite lattice and is by far the most abundant impurity detected within the lenses (as determined from ICP-OES by Polishchuk et $\mathrm{al}^{6}{ }^{6}$ ), it is reasonable to predict that the distribution of $\mathrm{Mg}$ is complementary to that of $\mathrm{Ca}$, as represented by the dashed red line in Fig. 1a. Besides the oscillations, the signal undergoes a decrease from the sample's surface toward the bulk, which can be attributed to sample self-absorption. Moreover, the oscillations, although irregular, demonstrate a periodicity of $0.7-1.5 \mu \mathrm{m}$. These periodicity values coincide with the values determined from the nano-holotomographic (nano-HT) reconstruction of a slice from another lens sample (Fig. 1b). In a previous study by Dai et al. ${ }^{20}$, where $\mathrm{Mg}$-calcite was crystalized from polymerinduced liquid precursor (PILP), transition bars were observed and shown to be rich in the biopolymer that stabilizes the PILP. In light of this, we considered whether our layers are comprised of organics as well. The first experiment we performed to answer this question was recharacterization of the lenses by nano-HT after heat treatment for $70 \mathrm{~min}$ at $300^{\circ} \mathrm{C}$. Interestingly, same oscillations were observed in the nano-HT reconstruction of a lens as compared to those without annealing (Supplementary Fig. 1). We want to stress that the annealing temperature is sufficiently high for occluded proteins to decompose, as we have shown extensively in the past ${ }^{2,21,22}$, confirming that organic matter could not be solely responsible for the observed layered structure. Another strong indication that eliminates the possibility that the observed layers are comprised of higher organic concentration is the fact that these layers demonstrate smaller dspacings than the matrix, as we have previously shown ${ }^{6}$. This therefore contradicts the possibility of higher concentration of intracrystalline organics within the layers, which have been shown to induce expansion of the d-spacings rather than contraction $^{2,21,22}$. In this context, we measured the amount of occluded proteins in the brittle star lenses by means of amino acids analysis (AAA). We found that the brittle star lenses contain as little as $0.001 \mathrm{wt} \%$ of occluded proteins, as determined by AAA (Supplementary Table 1) (for comparison, the mature sea urchin spines contain $0.02 \mathrm{wt} \%$ occluded proteins ${ }^{23}$ ). This extremely low amount, together with the other above characterization results, clearly imply that the distribution of $\mathrm{Mg}$-rich nanoprecipitates (rather than the presence of proteins) is the most probable cause for the observed layered structure, although we do not rule out some possible synergistic effect involving organic matter in the formation of the layers.

We could confirm that the observed layered structure corresponds to a layered distribution of high Mg-particles using high-resolution transmission electron microscopy (HRTEM). Indeed, imaging of a lens' cross-section clearly shows a layered distribution of nanoparticles (Fig. 2). The image of Fig. 2 was acquired in high-angle annular dark-field scanning transmission electron microscopy mode (HAADF-STEM), which is sensitive to elemental composition (Z-contrast). The fact that the nanoparticles are darker contrast than the matrix implies that they are lighter, corresponding to the fact that they are richer in $\mathrm{Mg}$. Therefore, the particles seen in Fig. 2 correspond to the previously established coherent high Mg-nanoparticles, and they are organized in layers parallel to the surface. The particle-rich layers are irregularly spaced and irregularly packed with particles, explaining the irregular line profiles obtained with the different characterization techniques (Fig. 1, Supplementary Figs. 1, 2 and 4).

We would like to note that the difference in appearance of the layered structure between the different characterization techniques is due to the difference in length scales that they characterize. Indeed, the small thickness of the particle-rich layers (few tens of $\mathrm{nm}$ ) as seen in HRTEM cannot be imaged and detected in lower resolution techniques, and the micron-scale oscillations as seen in tomography are integrations of several thin layers, and cannot be detected at the high magnification used in HRTEM.

Additional evidence for the layered structure emerges when employing electron microscopy using a Z-sensitive backscattered electron (BSE) detector. For this examination, we embedded DAPs into epoxy resin and polished them to expose a section perpendicular to the lenses' surface. The acquired images indeed displayed oscillations in contrast, confirming the layered structure in the lenses (Fig. 3a). The stripes exhibiting darker contrast in Fig. 3 correspond to layers that comprise relatively higher concentrations of $\mathrm{Mg}$-rich nanoparticles (therefore, less dense, being richer in $\mathrm{Mg}$ that is a lighter element as compared to $\mathrm{Ca}$ ). On the other hand, the stripes with brighter contrast correspond to layers that comprise relatively lower concentrations of Mg-rich nanoparticles (denser, being less rich in $\mathrm{Mg}$ ). To ensure that oscillations are present not only at the imaged surface but also in the bulk, we ion-milled an area of the sample using a focused ion beam (FIB). Continuing oscillations in density can be observed in the ion-milled portion (Fig. 3b). Since milling exposes a previously hidden surface, the observed oscillations are clear indications of the layered structure of the lenses, rather than the topography or the after-effects of sample preparation (polishing), a common concern in routine SEM preparations. The oscillations observed here are rather irregular (Fig. $3 \mathrm{a}, \mathrm{b}$ ) but range from 0.7 to $1.0 \mu \mathrm{m}$ (Supplementary Fig. 2), confirming the data on periodicity obtained from the nano-HT experiment (Fig. 1). 

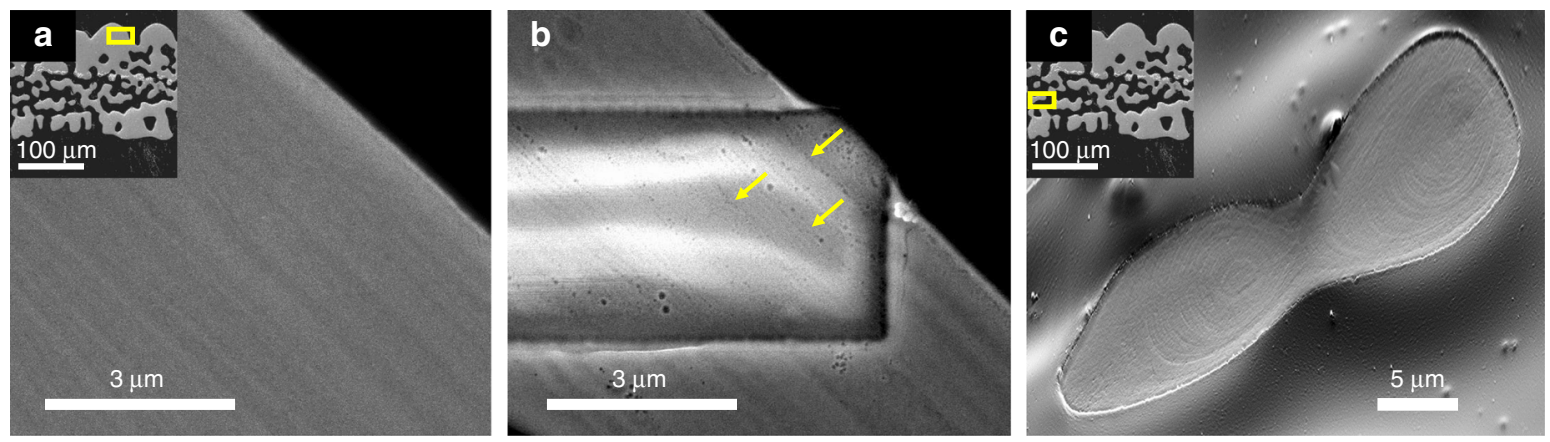

Fig. 3 The layered structure revealed by electron microscopy a HRSEM (BSE detector) image of a polished lens section. b BSE image of the same specimen after ion-milling in the FIB. Yellow arrows point at visible layers in the milled box. c HRSEM (secondary electron (SE) detector) images from polished DAP. The insets in $\mathbf{a}$ and $\mathbf{c}$ are a low magnification image of the polished DAP, the yellow rectangles represent the corresponding areas of interest
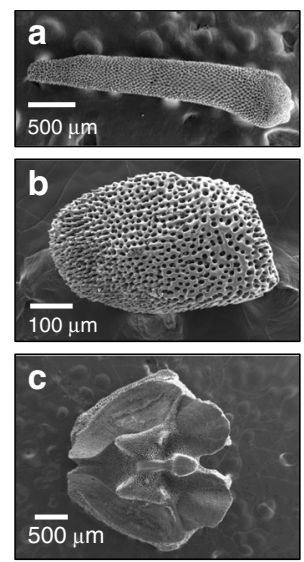

d

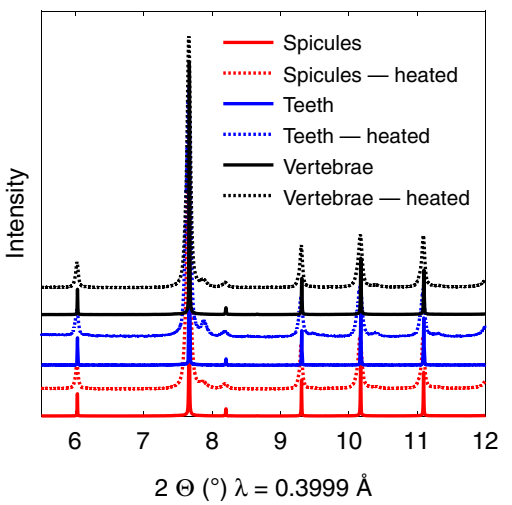

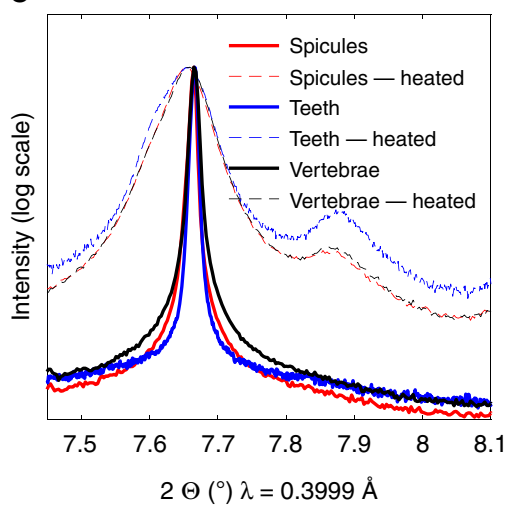

Fig. 4 Other calcitic parts' morphology and structure. a-c HRSEM images of the O. wendtii brittle star, showing a arm vertebrae, $\mathbf{b}$ spicules, and $\mathbf{c}$ teeth of the organism. d Diffractogram of these calcitic parts at room temperature and after heating treatment at $400{ }^{\circ} \mathrm{C}$. e Zoom-in on the (104) diffraction peaks plotted on logarithmic scale

High-resolution scanning electron microscope (HRSEM) images of all polished parts of the brittle star arm plates all show oscillations parallel to the surface (Fig. 3c), confirming the layered manner of its single-crystalline structure. In addition, the layered structure can be seen to appear as a series of periodic rings, probably resulting from the mechanism of its formation. The image in Fig. 3c was acquired using a secondary electron (SE) detector so the apparent contrast can originate from both topography and Z-contrast. We note that intensity oscillations originating from Z-contrast would attest for layers with different Mg-content, and oscillations originating from topography contrast in the polished sample would attest for layers with different polishing rate, which should be the case of layers with different content of Mg-rich particles. HRSEM image of a broken lens reveals similarly contrasting layers (Supplementary Fig. 3).

We found similar observations in other $O$. wendtii skeletal parts: spicules, arm vertebrae (both situated along the arms of the brittle star), and teeth (from the central ventral structure) (Fig. 4a-c). Synchrotron high-resolution powder X-ray diffraction (HRPXRD) patterns of the different crushed skeletal parts were identical and corresponded to single-phase Mg-calcite (Fig. 4d). In all elements, we observe the presence of the coherently aligned nanosized Mg-rich phase by demonstrating a broad hump at the base of the diffraction peaks plotted on the logarithmic scale and the emergence of a separate high- $\mathrm{Mg}$ calcite nanophase after the skeletal parts were heated at $400^{\circ} \mathrm{C}$ (Fig. 4e). The lattice parameters of the different phases can be obtained from the diffractogram by means of Rietveld refinements, and the amount of $\mathrm{Mg}$ present in the calcite can then be quantified from the lattice parameters using previously established equations ${ }^{24}$ (Supplementary Table 2).

Moreover, HRTEM imaging of an untreated section of a spicule shows a dark matrix with coherently incorporated bright nanodomains (Fig. 5a). These bright nanodomains grow and lose coherence after heat treatment at $400^{\circ} \mathrm{C}$ (Fig. 5b), akin to the behavior demonstrated by Polishchuk et al. in the case of the lenses ${ }^{6}$. Based on these findings, we conclude that the different calcitic skeletal parts of the brittle star (lenses, spicules, arm vertebrae and teeth) all have the same nanostructure, consisting of Mg-rich nanoparticles ( 40 mol\% $\mathrm{MgCO}_{3}$ ) coherently embedded in a relatively lower Mg-calcite matrix $\left(\sim 13 \mathrm{~mol} \% \mathrm{MgCO}_{3}\right)$, with resulting compressed matrix and overall improved toughness.

Nano-HT reconstructions of the spicules display contrasting layers identical to those in the lenses (Fig. 6), indicating that the layered distribution of Mg-rich nanoparticles is common to the organism's other calcitic parts as well, and is probably related to the formation mechanism of its skeletal parts. In Fig. 6, the bright layers are denser than the dark layers. Since the Mg-rich nanoparticles are coherent with the matrix, the lattice size does not change significantly between the layers, but there is a change in the elemental composition. Thus, the denser layers contain less $\mathrm{Mg}$ i.e., these are the layers that are depleted of $\mathrm{Mg}$-rich nanoparticles. The less dense layers contain more $\mathrm{Mg}$, i.e., they contain more Mg-rich nanoparticles. Intensity line plots of these reconstruction images reveal an oscillation periodicity of $\sim 1 \mu \mathrm{m}$ (Supplementary Fig. 4). 


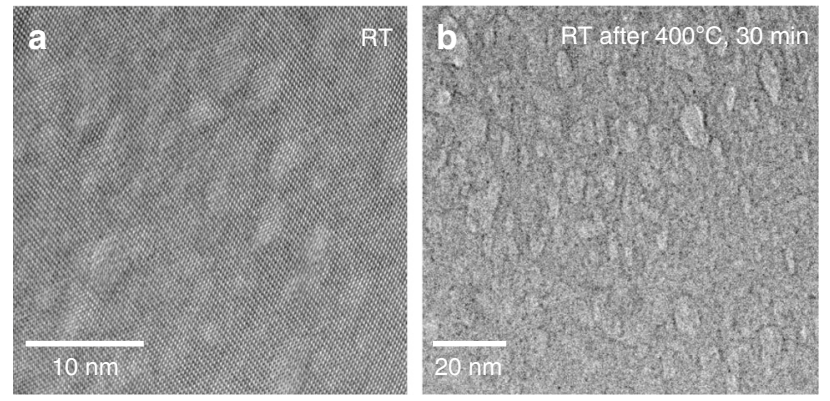

Fig. 5 Coherent nanoparticles in the brittle star spicules. a HRTEM image of a spicule, revealing brighter nanodomains, which are coherent with the matrix. b HRTEM image after in-situ heating at $400^{\circ} \mathrm{C}$, demonstrating growth of the bright nanodomains and loss of coherency
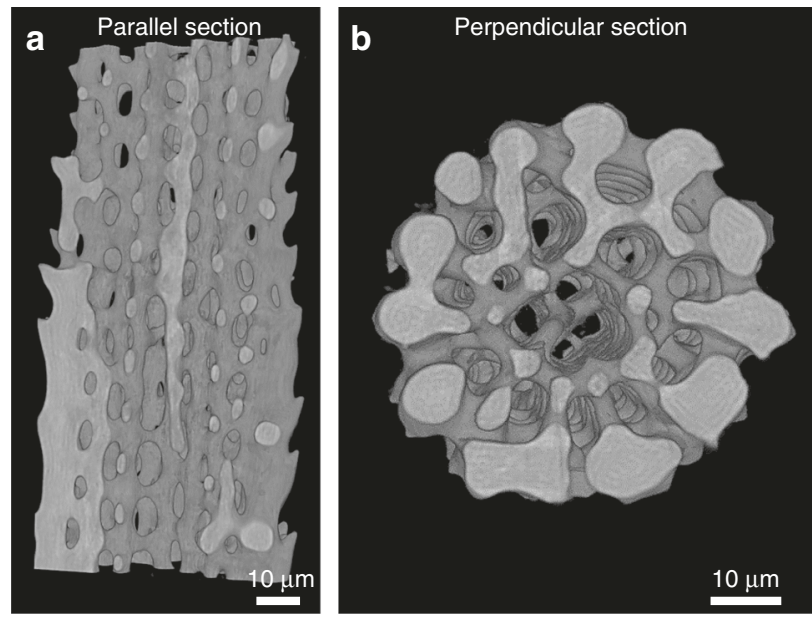

Fig. 6 3D morphology and layered structure of spicules. Nano-HT reconstructed parallel section (a) and horizontal section (b) of the brittle star spicule (pixel size is $120 \mathrm{~nm}$ )

Formation model. Considering the extreme complexity and efficiency of the brittle star's skeletal nanostructure, it is crucial to understand the mechanism of its formation. Some calcitic and aragonitic biominerals (such as mollusk shells ${ }^{25}$ or the sea urchin spicule $^{26}$, spine ${ }^{27}$, and teeth ${ }^{28}$ ) have been shown to be formed through the crystallization of an amorphous precursor ${ }^{25-29}$. That non-classical crystallization pathway, allows the formation of outof-equilibrium supersaturated solid solutions (such as high-Mg calcite) as well as the formation of intricate rounded crystal shapes ${ }^{29}$. Since this was also found to be the case with respect to the calcitic components of the brittle star, it is reasonable to assume that those components were formed via a Mg-rich amorphous calcium carbonate (Mg-ACC) precursor. The exact structure and even the state of the amorphous ACC precursor can vary ${ }^{30-33}$; the latter has indeed been shown to exist as a gel ${ }^{34}$ or as a liquid ${ }^{20,35-41}$. Notably, liquid ACC phase has been shown to be stabilized by the presence of acidic proteins, a process called polymer-induced liquid precursor (PILP) process ${ }^{20,38-41}$. It was moreover shown that a much lower amount of protein was necessary to induce the liquid amorphous precursor phase when $\mathrm{Mg}$ was present in the system ${ }^{41}$. Likewise, we assume that the amorphous precursor of the brittle star skeletal parts is in a gel or liquid-like state, possibly stabilized by both the $\mathrm{Mg}$ and the low amount of proteins present in the system. Below we present a model depicting the formation of the brittle star Mg-calcite nanostructure from an amorphous liquid or gel-like Mg-ACC precursor to a $\mathrm{Mg}$-calcite nanostructure containing periodic layers with varying concentrations of coherent $\mathrm{Mg}$-rich nanoinclusions. In this scenario, the initial stage is the liquid or gel-like supersaturated Mg-ACC precursor formed as a result of vital activity of the brittle star. Formation of the nanostructured $\mathrm{Mg}$ calcite then occurs in two steps. First, the Mg-ACC precursor undergoes a spinodal decomposition into Mg-rich nanodomains and a Mg-depleted ACC matrix. In the second step, this amorphous reorganized precursor crystallizes from a single nucleation point and through an advancing crystallization front that creates the layered structure of the Mg-rich nanodomains by the routes considered below.

First step: spinodal decomposition of $\mathrm{Mg}$-ACC precursor. Thermodynamic studies have shown that $\mathrm{Mg}$-calcite can be considered as similar to a binary solid solution of its two endmembers, calcite, and dolomite ${ }^{42,43}$. According to this approach, the free energy of mixing of $\mathrm{Mg}$-calcite can be written using the two-parameter representation of the Gibbs free energy of mixing: ${ }^{43}$

$$
\begin{gathered}
\Delta G\left(X_{\mathrm{D}}\right)=R T\left(X_{\mathrm{C}} \ln X_{\mathrm{C}}+X_{\mathrm{D}} \ln X_{\mathrm{D}}\right)+X_{\mathrm{D}}\left(1-X_{\mathrm{D}}\right) \\
{\left[A_{0}+A_{1}\left(2 X_{\mathrm{D}}-1\right)\right],}
\end{gathered}
$$

where $X_{\mathrm{C}}$ is the mole fraction of calcite and $X_{\mathrm{D}}$ is the mole fraction of dolomite, $X_{\mathrm{C}}=\left(1-X_{\mathrm{D}}\right)$, and $R$ is the gas constant. According to Busenberg and Plummer ${ }^{42}, A_{0}=12.6 \pm 0.2 \mathrm{~kJ} . \mathrm{mol}^{-1}$ and $A_{1}=$ $4.7 \pm 0.1 \mathrm{~kJ} \cdot \mathrm{mol}^{-1}$, as fitted from their experimental solubility results. The similar values of $A_{0}=12.4 \mathrm{~kJ} \cdot \mathrm{mol}^{-1}$ and $A_{1}=2.5 \mathrm{~kJ}$. mol $^{-1}$ were estimated by Lerman ${ }^{43}$ by extrapolating $A_{0}$ and $A_{1}$ values from high-temperature experimental compositions of $\mathrm{Mg}$ calcite phases ${ }^{44}$. Spinodal decomposition of this solution occurs if the second derivative of the free energy is negative:

$$
\frac{\partial^{2} G}{\partial X_{\mathrm{D}}^{2}}=\frac{R T}{X_{\mathrm{D}}\left(1-X_{\mathrm{D}}\right)}-2 A_{0}-6 A_{1}\left(2 X_{\mathrm{D}}-1\right)<0 .
$$

At room temperature and using the values of $A_{0}$ and $A_{1}$ constants from Busenberg and Plummer ${ }^{42}$, this condition is satisfied for $0.14<X_{\mathrm{Mg}}<0.48 \quad\left(X_{\mathrm{Mg}}=X_{\mathrm{D}} / 2\right) \quad$ (Supplementary Fig. 5). The occurrence of spinodal decomposition is conditional on the diffusion of $\mathrm{Mg}$ and $\mathrm{Ca}$, which is too slow in solid magnesian calcites under ambient conditions but can be fast enough in liquids and gels. Here, we assumed that Mg-ACC solutions are gel or liquid-like and are thermodynamically similar to the corresponding crystalline solution. The presence of charged organic molecules in a Mg-ACC precursor may alter thermodynamic and kinetic parameters of the system. However, we believe that such low amounts of proteins as observed in brittle star influence mainly the kinetic parameters such as effective diffusion coefficients of $\mathrm{Mg}$ and $\mathrm{Ca}$ ions. The supersaturated $\mathrm{Mg}$ ACC could then be approximated by a similar binary solution with two end-members, amorphous $\mathrm{CaCO}_{3}$ and amorphous $\mathrm{Ca}_{0.5} \mathrm{Mg}_{0.5} \mathrm{CO}_{3}$, which-like magnesian calcite - can be unstable. Therefore, spinodal decomposition should occur for $0.14<X_{\mathrm{Mg}}<$ 0.48 , similarly to crystalline magnesium calcite. In contrast to solid magnesium calcites, spinodal decomposition of liquid or gel-like Mg-ACC is kinetically possible at room temperatures (see Supplementary Note 1). Moreover, we can expect that the MgACC precursor of brittle star lenses contains the same global amount of $\mathrm{Mg}$ as the lenses, being of $\eta=15.2 \mathrm{~mol} \%$ (with $\eta=$ $\mathrm{Mg} /(\mathrm{Ca}+\mathrm{Mg}))$, as obtained by Polishchuk et al. ${ }^{6}$ Therefore, at this $\mathrm{Mg}$ concentration, the above thermodynamic and kinetic considerations predict a spinodal decomposition of the gel-like Mg-ACC precursor. Thus, the hypothesis of spinodal decomposition of the Mg-ACC precursor to Mg-depleted calcite matrix and Mg-rich nanodomains is reasonable. We emphasize that the spinodal decomposition is indeed the most probable way to 
obtain numerous nanoparticles that are homogeneously distributed throughout the matrix. Other possibilities such as multiple nucleation of Mg-rich amorphous or crystalline particles from a metastable amorphous state are less likely from the kinetic point of view due to comparatively slow nucleation rates.

To support the hypothesis that the homogeneous $15-\mathrm{mol} \%$ $\mathrm{Mg}$-ACC is unstable by analogy with the $15-\mathrm{mol} \% \mathrm{Mg}$-calcite, we synthesized Mg-ACC by fast mixing of solutions of $\mathrm{CaCl}_{2}$ and $\mathrm{MgCl}_{2}$ with solutions of $\mathrm{Na}_{2} \mathrm{CO}_{3}$. The $\mathrm{Mg}$-ACC in solution can be analogous to a gel-like phase. We then crystallized the $\mathrm{Mg}$ ACC solutions by exposing them to mild hydrothermal conditions $\left(135^{\circ} \mathrm{C}, 3 \mathrm{bar}\right)$. This synthesis protocol is one of the protocols in which Mg-ACC is used as a precursor to the subsequently crystallized high- $\mathrm{Mg}$ calcite phases ${ }^{45,46}$. The outcome of the hydrothermal treatment is the fast crystallization of the Mg-ACC, which otherwise, in its amorphous state, is stable over time ${ }^{47}$. In this way, we synthesized $\mathrm{Mg}$-calcite powders from a series of Mg-ACC precipitates, which we had obtained using different concentrations of $\mathrm{Mg}$ in solution (see Experimental section). XRD of the synthesized powders indicated mixtures of $\mathrm{Mg}$-calcite and aragonite (see Supplementary Table 3 for the relative amounts of aragonite). The amount of $\mathrm{Mg}$ in the synthesized calcite can be visualized by the shift of the calcite phase in the diffractogram (Fig. 7), and can be quantified from the calcite lattice parameter values, which decrease with the amount of $\mathrm{Mg}$ present in the calcite phase ${ }^{24}$. From the XRD data it appears that intermediate Mg-calcites $(0.10<\eta<0.34)$ do not form; instead, the precipitates obtained are a mixture of higher and lower Mg-calcite phases and a large amount of aragonite. The absence of mid-Mg calcite $(0.10<\eta<0.34)$ when crystallized at moderately high temperatures indicates that a decomposition might have already occurred in the amorphous precursor. Indeed, since the diffusion is limited in the solid phase (even at $135^{\circ} \mathrm{C}$ ), the decomposition probably already occurs in the gel-like amorphous state rather than in the crystalline phase, thus supporting our assumptions.

We would like to note that the synthesis of high-Mg calcite is not trivial. Indeed, at ambient conditions, crystallization of $\mathrm{Mg}$ -

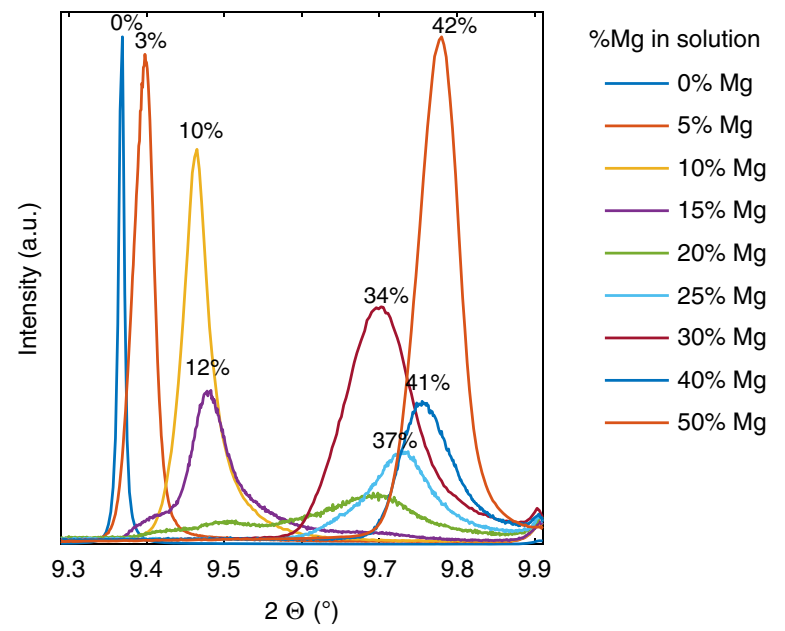

Fig. 7 Instability of $\mathrm{Mg}-\mathrm{CaCO}_{3}$ for a range of $\mathrm{Mg}$ composition. (104) XRD peak from $\mathrm{Mg}$-calcite crystallized from hydrothermally treated $\mathrm{Mg}-\mathrm{ACC}$ in solution. Data were acquired by synchrotron radiation $(\lambda=0.496 \dot{A})$.

Numbers near the peaks are the $\eta(\mathrm{Mg} / \mathrm{Ca}+\mathrm{Mg})$ in the calcite crystals, obtained from the refined lattice parameters and the equations linking the lattice parameters and $\eta$ obtained by Zolotoyabko et al. ${ }^{24}$. The diffractograms are normalized to the highest diffraction peaks (calcite (104) peak for the powders precipitated from solutions containing 0 or $50 \% \mathrm{Mg}$ in solutions, and aragonite peak for the other powders)
ACC does not form high-Mg calcite and aragonite is favored, unless some specific organic molecules are involved in the synthesis ${ }^{41,48}$. Here, we have established that an extremely low amount of proteins is present in the biomineral. Moreover, the above theoretical considerations rely on established models that do not involve organic matter. Therefore, although some biological control involving organic molecules certainly takes place, we believe that $\mathrm{Mg}$ is the key player in the $\mathrm{Mg}$-ACC spinodal decomposition process. To prove this point, we have chosen a protocol that isolates the effect of $\mathrm{Mg}$ amount, in the absence of organic additives (even though this protocol does not reproduce the biological ambient conditions of the biomineral formation). To further evaluate the effect of organic molecules on the behavior of Mg-ACC, we performed identical Mg-calcite syntheses, but in the presence of aspartic acid, a common amino acid involved in biomineralization. In these conditions, the intermediate-Mg calcite $(0.10<\eta<0.34)$ could form (Supplementary Fig. 6), revealing that these otherwise unstable Mg-ACC compositions can be stabilized by the presence of organic molecules. Additional syntheses, in the presence of polyaspartic acid, a polypeptide used to model the proteins involved in biomineralization ${ }^{38-41}$, reconfirmed this result (Supplementary Fig. 7). Therefore, we suggest that both the amount of $\mathrm{Mg}$ corresponding to $\mathrm{Mg}$-ACC instability range and the low amount of protein involved are specificities of the brittle star skeletal parts that enable spinodal decomposition of the Mg-ACC precursor, to Mg-rich domains and Mg-depleted matrix. This biomineral formation mechanism therefore uses the thermodynamic conditions created by its biological environment but this formation step is not directly biologically controlled; it can rather be explained by classical thermodynamics, a phenomenon that was shown in another biomineral, the prismatic layer P. Nobilis ${ }^{49,50}$.

Second step: crystallization and layered structure formation. The next step in the formation sequence is the crystallization of the decomposed Mg-ACC precursor.

The crystallization probably starts in the Mg-depleted matrix, since Mg-rich ACC is more stable against crystallization (Fig. $8 \mathrm{~d})^{47,51}$. We consider two possible crystallization routes leading to the formation of the layered structure (Fig. 8e-j). One possible route is depicted in Fig. 8e-g: The crystallization expands through an advancing crystallization front that faces a matrix containing high-Mg nanodomains, a scenario that can be comparable to the extensively studied case $e^{52-55}$ in which particles face a solidification front, on a larger length scale, such as in the case of freezing water-saturated soil ${ }^{53}$. Following the commonly accepted model for the latter situation, we can assume that the crystallization front and the particles are subjected to attractive and repulsive forces, which alternatively exceed one another, causing alternate embedding and repelling of the particles by the crystallization front. The disjoining force responsible for this repelling is still a matter of debate; it is usually assumed to result from van der Waals and electrostatic interactions between the dispersed nanoparticles and the solidification front. Here, we assume that a Columbian repulsive force exists between them, $F_{\mathrm{q}}$, maybe as a result of absorption of negatively charged organic and water molecules, both on the nanodomains and the crystallizing surface (the presence of these molecules at the crystallization front can result from organic molecules exclusion during crystallization $\left.{ }^{20}\right)$. The disjoining force is countered by an attractive force, the lubrication force $\left(F_{\mu}\right)$ that is associated with the flow of viscous fluid near the base of the dragged particle when repelled by the crystallization front ${ }^{54-56}$. This lubrication force depends on the viscosity of the suspension medium facing the crystallization front $(\mu$-itself depending on the volume fraction of the 


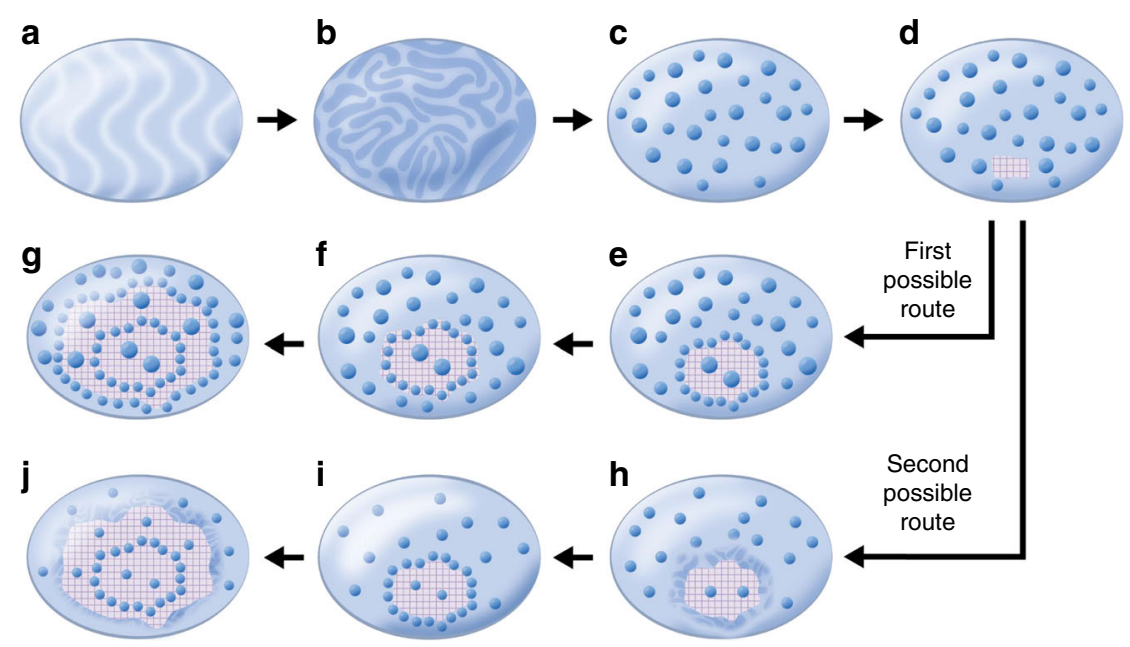

Fig. 8 Proposed mechanism of formation of layered structure. a Gel-like or liquid-like Mg-ACC precursor containing $15 \% \mathrm{MgCO}_{3}$. b Decomposition starts from growing fluctuations in concentration (spinodal decomposition of $\mathrm{Mg}$-ACC solution). c Large $\mathrm{Mg}$-concentration fluctuations result in formation of Mg-rich nanoparticles. d At some point, a nucleus of crystalline Mg-depleted calcite appears. e, $\mathbf{f}$ First possible crystallization route: e the crystalline nucleus grows through the gel, rejecting small particles (below a critical size) and incorporates large particles (above the critical size). $\mathbf{f}$ Local gel viscosity increases owing to the increase in volume fraction of the particles reaching the critical value at which particles are trapped and coherently crystallize. $\mathbf{g}$ The process is repeated. $\mathbf{h}$-j Second possible crystallization route: $\mathbf{h} \mathrm{Mg}$ exclusion from crystallizing calcite and $\mathrm{Mg}$ enrichment of the vicinal $\mathrm{Mg}$-ACC leads to secondary spinodal decomposition near the crystallization front. i Resulting formation of additional Mg-rich nanoparticles. $\mathbf{j}$ Advancement of crystallization front leading to formation of particle-enriched crystalline layer; thereafter the process of $\mathrm{Mg}$ exclusion and $\mathrm{Mg}$-ACC secondary spinodal decomposition is repeated

particles $\left.\phi^{56}\right)$ and on the size of the particles: $F_{\mu}(R, \mu(\phi))$. The transition from rejection to encapsulation of the particles is achieved when $F_{\mathrm{q}}=F_{\mu}$. This condition allows us to derive the maximum radius of rejected particles $R_{\mathrm{m}}(\mu)$. The larger particles $\left(R>R_{\mathrm{m}}(\mu)\right)$ are trapped by the crystalline front and encapsulated (or coherently crystallized in our case), while smaller particles $(R<$ $\left.R_{\mathrm{m}}(\mu)\right)$ are repelled and pushed to the gel. Our estimations yield a critical radius of repelled particles $R_{\mathrm{m}}\left(\mu_{0}\right)=5 \mathrm{~nm}$ (see Supplementary Note 2). Polishchuk et al. assessed the average diameter of the nanoparticles as $\sim 5 \mathrm{~nm}$ (meaning $\bar{r} \sim 2.5 \mathrm{~nm})^{6}$; therefore, almost all of the particles should be rejected by the crystallization front $\left(R<R_{\mathrm{m}}\left(\mu_{0}\right)\right)$. The crystallization front then sweeps up the $\mathrm{Mg}$-rich nanodomains while expanding radially (Fig. 8e). However, if the crystallization front repels a relatively high amount of nanoparticles, the volume fraction of the nanoparticles in proximity to the crystallization front will increase. The viscosity of the gel facing the crystallization front will then reach a critical value above which the lubrication force exceeds the repulsion force; the nanodomains will then not be repelled into the gel but will rather be pushed towards the crystallization front and forced to crystallize within the calcite matrix (Fig. 8f), creating a nanoparticle-rich crystalline layer. After incorporating the accumulated nanoparticles in the crystalline phase, the crystallization front will again face the lowered viscosity gel (with a low concentration of Mg-rich nanodomains). The process is then repeated, creating an alternating nanoparticle-rich/nanoparticlepoor layered structure (Fig. 8e-g).

An alternative or synergetic route leading to the oscillatory distribution of Mg-rich nanoparticles is depicted in Fig. 8h-j: This route relies on the phenomenon of impurity exclusion during ACC crystallization. ACC crystallization in the presence of polypeptides was indeed shown to form "transition bars", formed as a result of exclusion of the polymer impurity during liquid ACC crystallization ${ }^{20}$. Similarly, the crystallization of $\mathrm{Mg}$ depleted ACC matrix can be accompanied by the exclusion of $\mathrm{Mg}$ ions into a diffusion zone adjacent to the crystallization front. Thus, the crystallization front can be considered as a source of $\mathrm{Mg}$, and the adjacent Mg-ACC diffusion zone becomes enriched in Mg. Due to this enrichment, the Mg content in the vicinity of the crystallization front can exceed the critical Mg content above which the Mg-ACC matrix becomes unstable against spinodal decomposition (Fig. 8h). A new secondary spinodal decomposition will then take place and result in the formation of new $\mathrm{Mg}$ rich nanodomains. As a result, the density of Mg-rich nanodomains will be higher near the crystallization front (Fig. 8i). The advancement of crystallization front will then form a new crystalline layer with a higher concentration of Mg-rich nanodomains. This process is repeated, (Fig. 8j) creating the layered structure (see details in Supplementary Note 3).

\section{Discussion}

Previous studies of the $O$. wendtii brittle star lenses have revealed their excellent optical and mechanical properties as well as their elaborate nanostructure ${ }^{5,6}$, but how they form is largely unknown. In this study we observe the widespread finding that the coherent $\mathrm{Mg}$-rich nanoparticles previously discovered in the lenses ${ }^{6}$ also exist in other calcitic parts of this organism, namely the spicules, the teeth and the arm vertebrae. The Mg-rich nanoparticles are observed by using complementary X-ray and electron methods that proved that the Mg-rich particles are arranged in alternating nanoparticle-rich and nanoparticle-poor layers. Based on previous studies from the literature as well as experimental data on the newly discovered nanostructure of the brittle star's calcitic parts, we developed a scenario of its formation from a supersaturated gel or liquid-like Mg-ACC precursor. An important aspect of this scenario involves the instability of $\mathrm{Mg}$-ACC with $0.14<\eta<0.48(\eta=\mathrm{Mg} /(\mathrm{Ca}+\mathrm{Mg}))$, leading to spinodal decomposition of the 15\%-Mg-ACC precursor into $\mathrm{Mg}$ rich nanodomains and a Mg-depleted matrix. Crystallization of this decomposed precursor from a single nucleation point advances via the crystallization front through a suspension of $\mathrm{Mg}$ rich ACC, which results in the formation of single-crystal Mgcalcite with coherently embedded Mg-rich calcite nanoparticles distributed in alternating particle-enriched and particle-depleted layers. The spinodal decomposition within Mg-ACC has been 
supported by experimental findings in a synthetic assay. We also show that organics can alter the kinetics and suppress the spinodal decomposition. The finding that the mineralized parts of the brittle star comprise very low amounts of organics, as compared to that found in other organisms, suggests that this is a key parameter in the coherent nanoprecipitates formation via spinodal decomposition and may explain why this phenomenon was not observed to date in other organisms. The Mg-ACC instability discovered here is of fundamental importance, owing to the preponderance of $\mathrm{Mg}-\mathrm{ACC}$ in biominerals or as a precursor to biominerals. In future studies, proposed Mg-ACC stability model should be tested on different biominerals or synthetic systems.

\section{Methods}

O. wendtii collection and bleaching. Samples of $O$. wendtii were kindly provided by Dr. Gordon Hendler, Curator of the Echinoderm Department at the Natural History Museum of Los Angeles. The samples were collected in Belize during the 1980s and 1990s and preserved in AR grade ethanol. The arms of the brittle star were cut with a scalpel into segments and bleached in a solution of deionized (DI) water and sodium hypochlorite, with a 2:1 volume ratio of NaOCl:DI for $8 \mathrm{~h}$. To avoid dissolution of calcite in the liquid we added $2 \%\left(20\right.$ g.L $\left.\mathrm{L}^{-1}\right)$ sodium carbonate $\mathrm{Na}_{2} \mathrm{CO}_{3}$ to the DI water. After removal of the organics the solution was poured out, and the mineralized phase was rinsed several times with DI water and left to dry in the air.

AAA. Powdered sample was fully dissolved in $200 \mu \mathrm{L}$ of a $1 \mathrm{M} \mathrm{HCl}$ solution. DI water was added to complete $10 \mathrm{~mL}$ total. After hydrolysis, the sample was left to dry and then dissolved in DI water, a few $\mu \mathrm{L}$ were then taken to be measured and analyzed for amino acid.

HRSEM. Carbon coated samples were imaged with the Zeiss Ultra-Plus FEG-SEM, using accelerating voltages of $4-20 \mathrm{kV}$.

HRTEM. The FEI Titan 80-300 FEG-S/TEM system was used on FIB-cut crosssections, in bright-field TEM mode. The FEI Titan Cubed Themis G2 60-300 was used on a FIB-cut cross-section, in HAADF-STEM mode, at extremely low current of $7.5 \mathrm{pA}$ in order not to damage the samples.

Synchrotron HRXRD. Diffraction patterns of bleached powdered samples were acquired in the ID-22 beamline at the European Synchrotron Radiation Facility (ESRF), at wavelength of $0.399 \AA$ and $0.496 \AA$. The diffraction patterns were analyzed by Rietveld refinements using GSAS II software ${ }^{57}$.

Nano-HT. Nano-tomography datasets were obtained at the ID-16B beamline of the ESRF. Bleached samples of dorsal arm plates (DAPs) were sectioned with a scalpel, and small fragments containing several lenses were mounted on the tip of a metal needle and fixed with epoxy. To isolate a single lens, the small DAP fragment was fixed onto an automatic stage equipped with a $\mathrm{CO}_{2}$ laser that cut the superfluous parts in order to isolate one lens. The samples were positioned at four different distances between the focal plane of the X-ray beam, which focused down to a spot size of $50 \times 50 \mathrm{~nm}^{2}$ using two multilayer-coated Si mirrors in Kirkpatrick-Baez (KB) configuration operated under pink beam mode at $29.6 \mathrm{KeV}$ and $17.5 \mathrm{KeV}$, and the camera. While the sample rotated $\left(360^{\circ}\right), 2001$ high-resolution images were collected. The datasets were then first processed using a phase retrieval algorithm based on ESRF in-house software using the GNU Octave programming environment ${ }^{58}$. In a second step, 3D volumes using the filtered phase maps were reconstructed using back projection with the ESRF python software PyHST2 ${ }^{59}$.

XRF tomography. XRF tomography was also performed at the ID-16B beamline of the ESRF, with the same focusing optics. In this set-up, the sample was mounted on a rotating stage and horizontal line-scans were performed using the focused beam for each rotation interval until a $360^{\circ}$ rotation is complete. Measurements were performed at $17.5 \mathrm{KeV}$. The XRF-emitted signal was collected by detectors situated perpendicular to the incident beam, then fitted and processed using the Python Multi-Channel Analyzer (PyMCA) program ${ }^{60}$. Elemental sinograms from all detectors were combined and were then further reconstructed by use of the XRDUA software ${ }^{61}$ that uses an iterative algorithm. The obtained 2D elemental distribution slices were finally partially corrected for self absorption.

FIB. We used the FEI Helios NanoLab G3 UC Dual Beam FIB in our experiment. Ion-milling was performed utilizing high gallium milling currents and accelerating voltage for $1 \mathrm{~min} 40 \mathrm{~s}$ at $0.23 \mathrm{nA}$. These conditions allowed $\sim 400 \mathrm{~nm}$ milling depth, as measured from close-ups images of the cross-section of the milled box. Samples were embedded in an epoxy resin and polished using a Struers Rotopol-31+ RotoForce-4 polishing machine and successively fine diamond lapping films.

Mg-calcite syntheses. Solutions of $0.2 \mathrm{M} \mathrm{Na}_{2} \mathrm{CO}_{3}$ and solutions of $0.2 \mathrm{M} \mathrm{CaCl}_{2}$ and $\mathrm{MgCl}_{2}$ (with varying relative amount of $\mathrm{MgCl}_{2}$ ) were mixed, forming colloidal solutions of Mg-ACC in water. In some of the syntheses, L-aspartic acid $(0.1 \mathrm{M})$ and poly-aspartic acid $\left(200 \mu \mathrm{g} . \mathrm{mL}^{-1}, \mathrm{M} . \mathrm{W} .=2000-11,000\right)$ were added to the $\mathrm{Na}_{2} \mathrm{CO}_{3}$ solutions. The beakers were then placed in an autoclave, at $135^{\circ} \mathrm{C}, 3 \mathrm{bar}$, for $2 \mathrm{~h}$. The solutions were filtered, rinsed, and dried.

\section{Data availability}

The data that support the findings of the study are available from the corresponding author upon reasonable request.

Received: 24 March 2019 Accepted: 27 August 2019

Published online: 08 October 2019

\section{References}

1. Berman, A. et al. Intercalation of sea urchin proteins in calcite: study of a crystalline composite material. Science 250, 664-667 (1990).

2. Pokroy, B. et al. Anisotropic lattice distortions in biogenic calcite induced by intra-crystalline organic molecules. J. Struct. Biol. 155, 96-103 (2006).

3. Ma, Y., Cohen, S. R., Addadi, L. \& Weiner, S. Sea urchin tooth design: an "AllCalcite" polycrystalline reinforced fiber composite for grinding rocks. Adv. Mater. 20, 1555-1559 (2008).

4. Ma, Y. et al. The grinding tip of the sea urchin tooth exhibits exquisite control over calcite crystal orientation and $\mathrm{Mg}$ distribution. Proc. Natl Acad. Sci. USA 106, 6048-6053 (2009).

5. Aizenberg, J., Tkachenko, A., Weiner, S., Addadi, L. \& Hendler, G. Calcitic microlenses as part of the photoreceptor system in brittlestars. Nature 412, 819-822 (2001)

6. Polishchuk, I. et al. Coherently aligned nanoparticles within a biogenic single crystal: a biological prestressing strategy. Science (80-.) 358, 1294-1298 (2017).

7. Seknazi, E. \& Pokroy, B. Residual strain and stress in biocrystals. Adv. Mater 30, 1707263 (2018).

8. Currey, J. D. Hierarchies in biomineral structures. Science 2434, 2002-2003 (2003).

9. Long, X., Ma, Y. \& Qi, L. Biogenic and synthetic high magnesium calcite-a review. J. Struct. Biol. 185, 1-14 (2014).

10. Wegst, U. G. K., Bai, H., Saiz, E., Tomsia, A. P. \& Ritchie, R. O. Bioinspired structural materials. Nat. Mater. 14, 23-36 (2015).

11. Finnemore, A. et al. Biomimetic layer-by-layer assembly of artificial nacre. Nat. Commun. 3, 966 (2012).

12. Tang, Z., Kotov, N. A., Magonov, S. \& Ozturk, B. Nanostructured artificial nacre. Nat. Mater. 2, 413-418 (2003).

13. Kim, Y.-Y. et al. Tuning hardness in calcite by incorporation of amino acids Nat. Mater. 15, 903-910 (2016).

14. Hendley, C. T. et al. Mechanistic insights into diblock copolymer nanoparticle-crystal interactions revealed via in situ atomic force microscopy. J. Am. Chem. Soc. 140, 7936-7945 (2018)

15. Giosia, M. Di et al. Bioinspired nanocomposites: ordered 2D materials within a 3D lattice. Adv. Funct. Mater. 26, 5569-5575 (2016).

16. Li, H. et al. Calcite prisms from Mollusk shells (Atrina Rigida): swiss-cheeselike organic-inorganic single-crystal composites. Adv. Funct. Mater. 21, 2028-2034 (2011)

17. Stöhr, S., O’Hara, T. D. \& Thuy, B. Global diversity of Brittle stars (Echinodermata: Ophiuroidea). PLoS ONE 7, e31940 (2012).

18. Wilkie, I. C. Functional morphology of the arm spine joint and adjacent structures of the Brittlestar Ophiocomina nigra (Echinodermata: Ophiuroidea). PLoS ONE 11, e0167533 (2016).

19. Duffy, D. M. Coherent nanoparticles in calcite. Science 358, 1254-1255 (2017).

20. Dai, L., Cheng, X. \& Gower, L. B. Transition bars during transformation of an amorphous calcium carbonate precursor. Chem. Mater. 20, 6917-6928 (2008).

21. Pokroy, B., Fitch, A. \& Zolotoyabko, E. The microstructure of biogenic calcite a view by high-resolution synchrotron powder diffraction. Adv. Mater. 18, 2363-2368 (2006).

22. Zolotoyabko, E. \& Pokroy, B. Biomineralization of calcium carbonate: structural aspects. CrystEngComm 9, 1156 (2007).

23. Aizenberg, J., Hanson, J., Koetzle, T. F., Weiner, S. \& Addadi, L. Control of macromolecule distribution within synthetic and biogenic single calcite crystals. J. Am. Chem. Soc. 119, 881-886 (1997).

24. Zolotoyabko, E. et al. Differences between bond lengths in biogenic and geological calcite. Cryst. Growth Des. 10, 1207-1214 (2010).

25. Weiss, I. M., Tuross, N., Addadi, L. \& Weiner, S. Mollusc larval shell formation: amorphous calcium carbonate is a precursor phase for aragonite. J. Exp. Zool. 293, 478-491 (2002). 
26. Beniash, E., Aizenberg, J., Addadi, L. \& Weiner, S. Amorphous calcium carbonate transforms into calcite during sea urchin larval spicule growth. Proc. R. Soc. Lond. Ser. B Biol. Sci. 264, 461-465 (1997).

27. Politi, Y., Arad, T. \& Klein, E. Carbonate phase sea urchin spine calcite forms via a transient amorphous calcium carbonate phase. Spine (Phila. Pa. 1976) 1161, 1161-1165 (2011).

28. Killian, C. E. et al. Mechanism of calcite co-orientation in the sea urchin tooth. J. Am. Chem. Soc. 131, 18404-18409 (2009).

29. Addadi, L., Raz, S. \& Weiner, S. Taking advantage of disorder: amorphous calcium carbonate and Its roles in biomineralization. Adv. Mater. 15, 959-970 (2003).

30. De Yoreo, J. J. et al. Crystallization by particle attachment in synthetic, biogenic, and geologic environments. Science (80-.) 349, aaa6760 (2015).

31. Cartwright, J. H. E., Checa, A. G., Gale, J. D., Gebauer, D. \& Sainz-Díaz, C. I. Calcium carbonate polyamorphism and its role in biomineralization: how many amorphous calcium carbonates are there? Angew. Chemie Int. Ed. 51, 11960-11970 (2012).

32. Rieger, J. et al. Precursor structures in the crystallization/precipitation processes of $\mathrm{CaCO} 3$ and control of particle formation by polyelectrolytes. Faraday Discuss. 136, 265-277 (2007).

33. Gebauer, D. \& Wolf, S. E. Designing solid materials from their solute state: a shift in paradigms toward a holistic approach in functional materials chemistry. J. Am. Chem. Soc. 141, 4490-4504 (2019).

34. Wolf, S. L. P., Caballero, L., Melo, F. \& Cölfen, H. Gel-like calcium carbonate precursors observed by in situ AFM. Langmuir 33, 158-163 (2017).

35. Wolf, S. E. et al. Carbonate-coordinated metal complexes precede the formation of liquid amorphous mineral emulsions of divalent metal carbonates. Nanoscale 3, 1158-1165 (2011).

36. Wallace, A. F. et al. Supersaturated CaCO 3 Solutions. Science (80-.) 692, 885-889 (2013).

37. Gebauer, D., Kellermeier, M., Gale, J. D., Bergström, L. \& Cölfen, H. Prenucleation clusters as solute precursors in crystallisation. Chem. Soc. Rev. 43, 2348-2371 (2014).

38. Bewernitz, M. A., Gebauer, D., Long, J., C€, H. \& Gower, L. B. A metastable liquid precursor phase of calcium carbonate and its interactions with polyaspartate. Faraday Discuss. 159, 23-25 (2012).

39. Gower, L. B. \& Odom, D. J. Deposition of calcium carbonate films by a polymer-induced liquid-precursor (PILP) process. J. Cryst. Growth $\mathbf{2 1 0}$ 719-734 (2000).

40. Olszta, M. J., Odom, D. J., Douglas, E. P. \& Gower, L. B. A new paradigm for biomineral formation: mineralization via an amorphous liquid-phase precursor. Connect. Tissue Res. 44, 326-334 (2003).

41. Cheng, X., Varona, P. L., Olszta, M. J. \& Gower, L. B. Biomimetic synthesis of calcite films by a polymer-induced liquid-precursor (PILP) process: 1 . Influence and incorporation of magnesium. J. Cryst. Growth 307, 395-404 (2007)

42. Busenberg, E. \& Niel Plummer, L. Thermodynamics of magnesian calcite solid-solutions at $25^{\circ} \mathrm{C}$ and 1 atm total pressure. Geochim. Cosmochim. Acta 53, 1189-1208 (1989).

43. Lerman, A. Paleoecological problems of $\mathrm{Mg}$ and $\mathrm{Sr}$ in biogenic calcites in light of recent thermodynamic data. Geochim. Cosmochim. Acta 29, 977-1002 (1965).

44. Goldsmith, J. R. \& Heard, H. C. Subsolidus phase relations in the system $\mathrm{CaCO}_{3}-\mathrm{MgCO}_{3}$. J. Geol. 69, 45-74 (1961).

45. Graf, D. L. \& Goldsmith, J. R. Some hydrothermal syntheses of dolomite and protodolomite. J. Geol. 64, 173-186 (1956).

46. Yang, H., Chai, S., Zhang, Y. \& Ma, Y. A study on the influence of sodium carbonate concentration on the synthesis of high $\mathrm{Mg}$ calcites. CrystEngComm 18, 157-163 (2016).

47. Loste, E., Wilson, R. M., Seshadri, R. \& Meldrum, F. C. The role of magnesium in stabilising amorphous calcium carbonate and controlling calcite morphologies. J. Cryst. Growth 254, 206-218 (2003).

48. Long, X., Ma, Y. \& Qi, L. In vitro synthesis of high Mg calcite under ambient conditions and its implication for biomineralization process. Cryst. Growth Des. 11, 2866-2873 (2011).

49. Bayerlein, B. et al. Self-similar mesostructure evolution of the growing mollusc shell reminiscent of thermodynamically driven grain growth. Nat. Mater. 13, 1102-1107 (2014).

50. Sommerdijk, N. A. J. M. \& Cusack, M. Crystals competing for space. Nat. Mater. 13, 1078-1079 (2014)

51. Politi, Y. et al. Role of magnesium ion in the stabilization of biogenic amorphous calcium carbonate: a structure-function investigation. Chem. Mater. 22, 161-166 (2010)

52. Uhlmann, D. R., Chalmers, B. \& Jackson, K. A. Interaction between particles and a solid-liquid interface. J. Appl. Phys. 35, 2986-2993 (1964).
53. Rempel, A. W., Wettlaufer, J. S. \& Worster, M. G. Premelting dynamics in a continuum model of frost heave. J. Fluid Mech. 498, 227-244 (2004).

54. Aubourg, P. F. Interaction of second-phase particles with a crystal growing from the melt (Massachusetts Institute of Technology, 1978).

55. Saint-Michel, B., Georgelin, M., Deville, S. \& Pocheau, A. Interaction of multiple particles with a solidification front: from compacted particle layer to particle trapping. Langmuir 33, 5617-5627 (2017).

56. Vand, V. Theory of viscosity of concentrated suspensions. Nature 155, 364-365 (1945).

57. Toby, B. H. \& Von Dreele, R. B. GSAS-II: the genesis of a modern open-source all purpose crystallography software package. J. Appl. Crystallogr 46, 544-549 (2013).

58. Cloetens, P. et al. Holotomography: quantitative phase tomography with micrometer resolution using hard synchrotron radiation x rays. Appl. Phys. Lett. 75, 2912-2914 (1999).

59. Mirone, A., Brun, E., Gouillart, E., Tafforeau, P. \& Kieffer, J. The PyHST2 hybrid distributed code for high speed tomographic reconstruction with iterative reconstruction and a priori knowledge capabilities. Nucl. Instruments Methods Phys. Res. Sect. B Beam Interact. Mater. Atoms 324, 41-48 (2014).

60. Solé, V. A., Papillon, E., Cotte, M., Walter, P. \& Susini, J. A multiplatform code for the analysis of energy-dispersive X-ray fluorescence spectra. Spectrochim. Acta Part B At. Spectrosc. 62, 63-68 (2007).

61. De Nolf, W., Vanmeert, F. \& Janssens, K. XRDUA: crystalline phase distribution maps by two-dimensional scanning and tomographic (micro) Xray powder diffraction. J. Appl. Crystallogr 47, 1107-1117 (2014).

\section{Acknowledgements}

This project has partially received funding from the European Union Horizon 2020 research and innovation program under the Marie Skłodowska-Curie grant agreement no. 642976-NanoHeal Project. We are grateful to Dr Yaron Kaufman for his help collecting HRTEM data. We acknowledge the European Synchrotron Radiation Facility for provision of synchrotron radiation facilities at ID22 and ID16B.

\section{Author contributions}

E.S. and S.K. have contributed equally to this work. E.S. and S.K. have performed the experiments, A.K. has developed the model, E.S., I.P., A.K. and B.P. have participated to the redaction of the manuscript, C.D. has helped with the XRD experiment, P.Z., N.B.S. J.V. and J-P.S. have helped with the tomography experiments and analysis. All authors have read and approved the manuscript. B.P. provided supervision of the research.

\section{Additional information}

Supplementary Information accompanies this paper at https://doi.org/10.1038/s41467019-12168-8.

\section{Competing interests: The authors declare no competing interests.}

Reprints and permission information is available online at http://npg.nature.com/ reprintsandpermissions/

Peer review information Nature Communications thanks Laurie Gower and other, anonymous, reviewers for their participation in the peer review of this work.

Publisher's note Springer Nature remains neutral with regard to jurisdictional claims in published maps and institutional affiliations.

Open Access This article is licensed under a Creative Commons Attribution 4.0 International License, which permits use, sharing, adaptation, distribution and reproduction in any medium or format, as long as you give appropriate credit to the original author(s) and the source, provide a link to the Creative Commons license, and indicate if changes were made. The images or other third party material in this article are included in the article's Creative Commons license, unless indicated otherwise in a credit line to the material. If material is not included in the article's Creative Commons license and your intended use is not permitted by statutory regulation or exceeds the permitted use, you will need to obtain permission directly from the copyright holder. To view a copy of this license, visit http://creativecommons.org/ licenses/by/4.0/.

(C) The Author(s) 2019 